\title{
Hieratische Fragmente des „Buches vom Fayum“ und ein Nachtrag zu BF Carlsberg
}

\author{
Hierzu Tafel I-III
}

Wie schon in ZÄS 122 angekündigt, sollen hier ein paar Fragmente des „Buches vom Fayum“ publiziert werden, die mir bei der Abfassung der Monographie über diesen Text noch nicht bekannt waren ${ }^{1}$. Es handelt sich dabei um drei Carlsberg-Papyri und um ein Fragment aus der Berliner Papyrussammlung, alle aus der römischen Zeit in Ägypten, sowie um eine Ergänzung zum illustrierten hieroglyphischen „Buch vom Fayum“ in Kopenhagen (BF Carlsberg 56) 2 . Die Reihenfolge der Behandlung der hieratischen Fragmente im nachfolgenden Text ergibt sich aus der Zeilenzählung im „Buch vom Fayum“. Im nachfolgenden Aufsatz geht es primär um die Bestimmung der Papyrusfragmente aus Berlin und Kopenhagen und nicht um den Inhalt des „Buches vom Fayum“. Für inhaltliche Fragen verweise ich auf die oben zitierte Publikation. Da es sich bei den zu bearbeitenden Fragmenten um solche in hieratischer Schrift handelt, scheint es mir folgerichtig zu sein, den hieratischen Schriftzeichen die Parallele ebenfalls in Hieratisch entgegenzustellen, auch wenn die dafür notwendigen Abbildungen auf den ersten Blick etwas aufwendig erscheinen. Jede Zeile aus den zu bearbeitenden Papyrusfragmenten wird - wie in der schon genannten Monographie - mit allen Parallelen dargestellt, mit dem Unterschied, daß hieratisch geschriebene Parallelen auch so wiedergegeben sind ${ }^{3}$. Um die Abbildungen nicht zu groß werden zu lassen, sind von den bereits bekannten Texten nur die unmittelbaren parallelen Textbereiche und ihre nähere Umgebung berücksichtigt ${ }^{4}$. Durch das Nebeneinanderstellen der hieratischen Texte soll auch gezeigt werden, wie nahe doch die Handschriften miteinander verwandt sind. Sieht man einmal von der sehr schwungvollen Handschrift „Botti G“ ab, so hat man den Eindruck, daß sie alle aus der gleichen Schule stammen, auch wenn natürlich für eine fundierte Aussage in diese Richtung weitere paläographische Untersuchungen notwendig wären. Da nun die Handschrift „Botti A“ in ihrem Kolophon auf den 5. September 135 n. Chr. datiert ist, halte ich eine Datierung der anderen Handschrift in etwa diese Zeit für sehr wahrscheinlich ${ }^{5}$.

1 Horst Beinlich, Das Buch vom Fayum (ÄA 51), 1991.

2 Für Hilfen und Hinweise danke ich Frau Bente Holmen, Frau Ingeborg Müller, Herrn Kim Ryholt und Herrn Karl-Theodor Zauzich. Das Photo des Berliner Papyrusfragments stammt von Frau Margarete Büsing.

. ${ }^{3}$ Abkürzungen: Die Abkürzungen entsprechen denen, die ich schon in meiner Arbeit „Das Buch vom Fayum“ verwendet habe: $\mathrm{BF}-$ Zeile $=$ Zeilenzählung innerhalb der genannten Arbeit für den ägyptischen Text B/H/A = Boulaq $/$ Hood $/$ Amherst, d. i., die Bezeichung für den grundlegenden hieroglyphischen Text des „Buches vom Fayum“. Botti = die hieratischen Textfragmente, die von Giuseppe Botti 1959 in seiner Arbeit „La glorificazione di Sobk e del Fayyum in un papiro ieratico da Tebtynis (Analecta Aegyptiaca) " publiziert worden sind.

${ }_{5}^{4}$ Auch hier sei bei weiterreichendem Interesse auf die Monographie verwiesen.

5 Damit soll aber nichts über die Datierung des „Buches vom Fayum“ als Komposition oder gar über einzelne Textteile gesagt sein. Das älteste datierbare Textfragment stammt aus der Zeit Augustus/Tiberius und befindet sich im Tempel von Kom Ombo (s. dazu „Buch vom Fayum“, S. 64 f.). Merkwürdigerweise drehten sich die Reaktionen, die ich zu meiner Publikation bekommen habe, meist um die Frage, ob der Text griechisch oder römisch ist. Ich halte diese Frage bei unserem gegenwärtigen Kenntnisstand für unwichtig. Wir haben in dem Kom Ombo-Text aus 
Die Texte auf den Rückseiten der Papyrusfragmente Carlsberg sind nicht Gegenstand des vorliegenden Aufsatzes, sie werden nur der Vollständigkeit halber mit abgebildet.

\section{1. pCarlsberg 310 (ehem. ICPCP 10.782), s. Taf. I}

Bei dem Papyrus handelt es sich um ein Fragment von 3,1 cm Breite und 3,7 cm Höhe, das recto und verso beschriftet ist. Recto erkennt man die Reste von 5 hieratischen Zeilen aus dem „Buch vom Fayum“, verso 5 Zeilen eines demotischen Textes, den man aber zum Beschriften so gedreht hat, daß die Oberkante des hieratisch beschrifteten Papyrus zur Unterkante des demotischen Papyrus wurde. Damit gleicht er dem weiter unten zu besprechenden pCarlsberg 311, und man kann nicht ausschließen, daß die beiden Papyri, wenn auch weit voneinander entfernt, von der gleichen Papyrusrolle stammen ${ }^{6}$. Eine Linierung ist weder auf der Vorder- noch auf der Rückseite des Papyrus festzustellen. Der hieratische Text stammt aus dem 1. Abschnitt des „Buches vom Fayum“, der hieratisch bisher nur in dem Textfragment „Botti F“ bekannt war.

Obwohl das Fragment recht klar lesbar zu sein scheint, macht es bei der genauen Einordnung in den Text des „Buches vom Fayum“ sehr viel Schwierigkeiten: Die dritte Zeile (s. Abb. 3) ist am klarsten zu lesen: spd.t ' nq.t. Ohne Zweifel ist diese Zeile eine Parallele zu der BF-Zeile 77. Hier beginnen aber bereits die Probleme. Wie aus dem hieroglyphischen Text ${ }^{7}$ zu ersehen ist, sind die Götternamen bei den Darstellungen am oberen bzw. unteren Bildrand zweimal genannt, einmal im Text selbst und einmal als Beischrift zur Darstellung. Im hieratischen Text wird diese Reihenfolge des hieroglyphischen Textes beibehalten. Es steht erst der Gottesname, und dann folgt, mit s.t tn eingeleitet, die Legende, in der der Gottesname abermals erscheint. Es stellt sich also die Frage, welche Nennung von Satet und Anuket wir hier haben. In der hieratischen Parallele (Botti F) scheint der einleitende Gottesvermerk entgegen der Lesung Bottis doch eher Anuket zu heißen, nicht Isis ${ }^{8}$.

Die gleiche Schwierigkeit bietet die darüberliegende zweite Zeile (s. Abb. 2). Auch hier finden wir einen Gottesnamen, nämlich den des Bockes von Mendes. Die Lesung selbst ist fast unproblematisch, wenn man einmal von der kleinen Lücke rechts von $\underline{d} d . t$ absieht, in der wohl ein zu erwartendes $n b$ nicht gestanden haben kann. Auch hier ist die Zuordnung - Anfang oder Ende der BF-Zeile 70 - offen. So nebensächlich diese Frage zunächst erscheint, so wichtig ist sie für die Zuordnung der anderen Zeilen, da man annehmen darf, daß untereinander stehende Textstellen etwa den gleichen Textabstand voneinander haben, den man mit Hilfe der Parallelen bestimmen kann. Da in der ersten Zeile der Rest eines hieratischen Zeichens mit einer Unterlänge in Form von zwei Beinen zu stehen scheint (s. Abb. 1), könnte die ungefähre Stelle des Textes, an der man dieses Zeichen zu suchen hat, bei der Identifizierung helfen. Unterstellt man nun, daß es sich bei beiden Nennungen des Bockes von Mendes um die vor den eigentlichen Text gesetzte Beischrift zur Darstellung handelt, bei der Nennung von Satet und Anuket aber um die

\footnotetext{
der früheren römischen Zeit ein Zeugnis, das nahe an der Grenze zwischen „griechisch“ und „römisch“ einzuordnen ist. Man darf also wohl vermuten, daß in die Endfassung des Textes, von der immerhin nun 20 Fragmente bekannt sind, zumindest vorrömisches Gedankengut eingeflossen ist. Die Frage aber, wie alt diese Gedanken wirklich sind und wann sie in die schriftliche Form gebracht wurden, die wir kennen, bleibt z. Zt. unbeantwortbar.

6 Ein ebenso gedrehter Papyrus findet sich unter den pCarlsberg noch mit der ICPCP-Nr. 10.781. Eine Zuordnung des recto dieses Papyrus zum „Buch vom Fayum“ war mir aber nicht möglich, obwohl einige der Wortfragmente durchaus zu diesem Text passen könnten.

7 S. dazu „Buch vom Fayum“, S. 142 bis 146 u. Taf. 2 bis 5.

8 Wie ich in meiner Publikation des „Buches vom Fayum“ einleitend angemerkt habe (S. 137), wurde die Lesung Bottis nicht grundsätzlich, sondern nur bei begründetem Zweifel überprüft. Es ist deshalb durchaus auch weiterhin möglich, daß sich Lesungsvorschläge Bottis anhand neuer Paralleltexte als zu revidieren erweisen werden. Der großartigen Leistung von Botti tut das keinen Abbruch.
} 


\section{IIIra tofy \\ ziate \\ ix $\rightarrow$ in \\ (3) 5 -3 \\ trits.}

recto

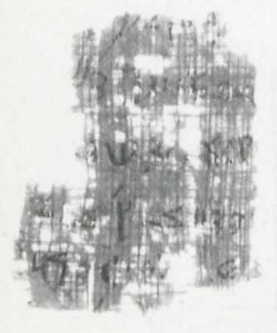

verso

pCarlsberg 310

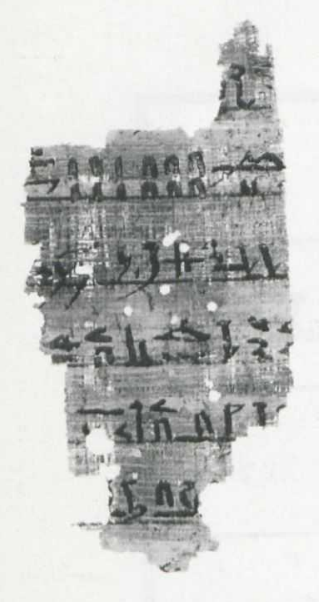

pBerlin 14473 b

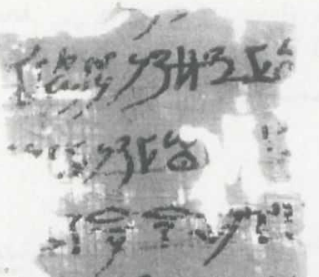

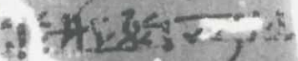
Atiricat?

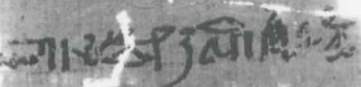

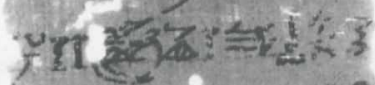

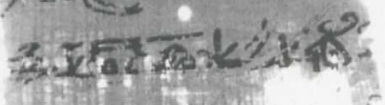

$\sin 2 x=$

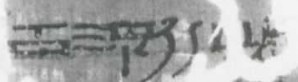

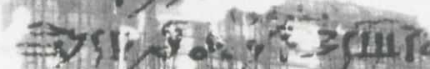
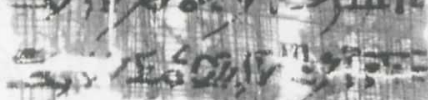

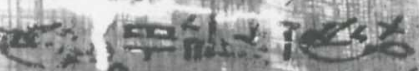

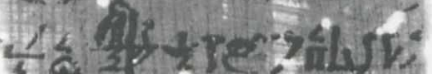

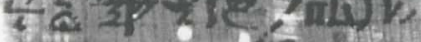
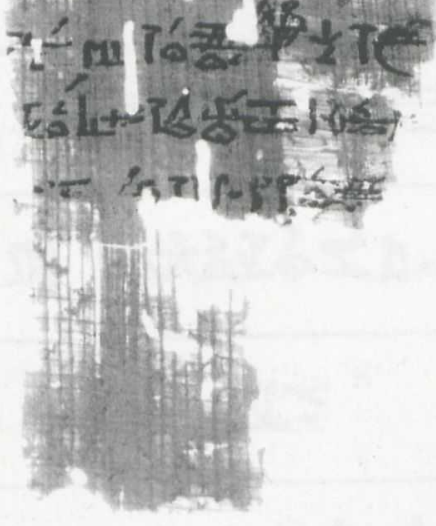

recto

pCarlsberg 311

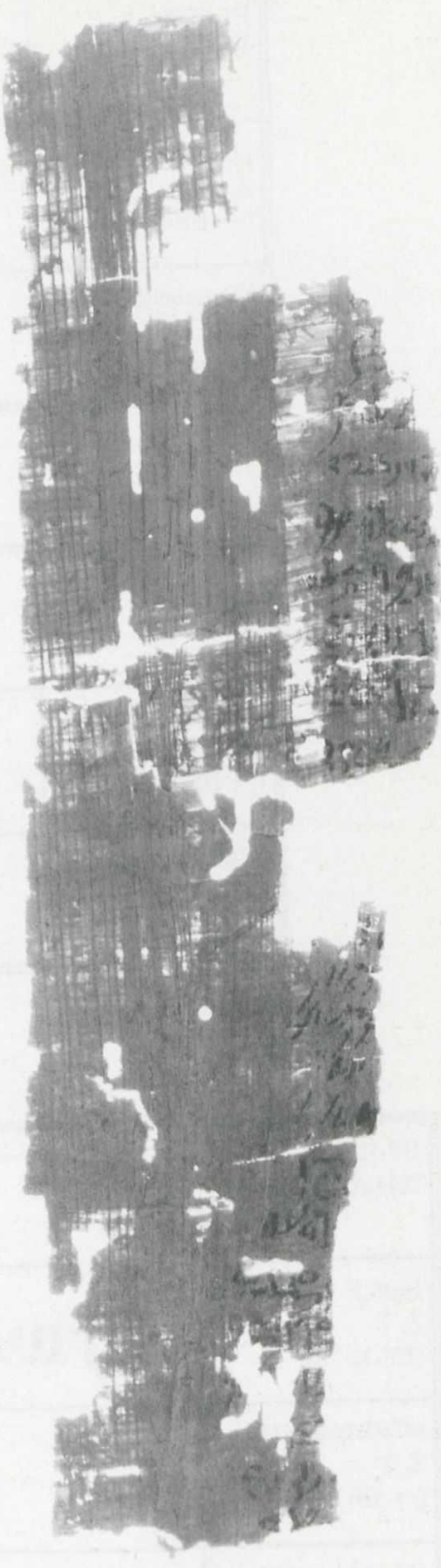

verso 


\begin{tabular}{|c|c|}
\hline $\begin{array}{l}\text { BF-Z. } 61 \\
\text { B/H/A }\end{array}$ & 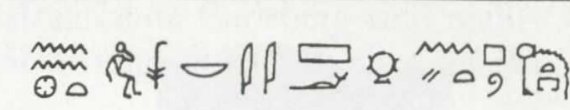 \\
\hline $\begin{array}{l}\text { pCarlsberg } 310 \\
\text { Z. } 1 \\
(\rightarrow \text { BF-Z. 62a) }\end{array}$ & a in \\
\hline $\begin{array}{l}\text { pCarlsberg } 310 \\
\text { Z. } 1 \\
\text { (Umschrift) }\end{array}$ & 经 \\
\hline
\end{tabular}

Abb. 1

\begin{tabular}{|l|r|}
\hline $\begin{array}{l}\text { BF-Z. 70 } \\
\text { B/H/A }\end{array}$ & \\
\hline $\begin{array}{l}\text { pCarlsberg 310 } \\
\text { Z. 2 } \\
\rightarrow \text { BF-Z. 72a) }\end{array}$ & \\
\hline $\begin{array}{l}\text { pCarlsberg 310 } \\
\text { Z. 2 } \\
(\text { Umschrift })\end{array}$ & \\
\hline
\end{tabular}

Abb. 2

\begin{tabular}{|c|c|}
\hline $\begin{array}{l}\text { BF-Z. } 77 \\
\text { B/H/A }\end{array}$ & 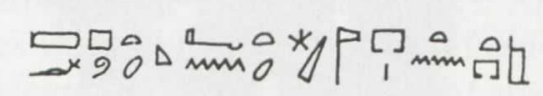 \\
\hline $\begin{array}{l}\text { Botti F } \\
\text { Z. } 7 \\
\text { (BF-Z. 78) }\end{array}$ & 'Il \\
\hline $\begin{array}{l}\text { pCarlsberg } 310 \\
\text { Z. } 3 \\
(\rightarrow \text { BF-Z. } 78 \text { a) }\end{array}$ & antes \\
\hline $\begin{array}{l}\text { pCarlsberg } 310 \\
\text { Z. } 3 \\
\text { (Umschrift) }\end{array}$ & 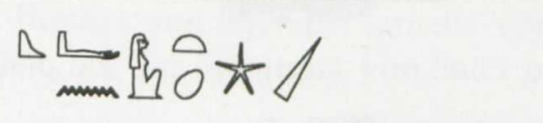 \\
\hline
\end{tabular}

Abb. 3

Nennung im Text, und überträgt den so gewonnenen Textabstand auf die Zeile Nr. 1, so kommt man in der BF-Zeile 61 in die Umgebung der Schreibung nn-nswt (Herakleopolis). Da pCarlsberg 310 hier, wie gesagt, ein Zeichen mit Unterlänge hat, darf man deshalb annehmen, daß es sich hier um das Zeichen des „sitzenden Kindes“ (Möller, Paläographie Nr. 30) handelt. Folgt man den genannten Zuordnungen der Zeilen 2 und 3 nicht, so trifft man auch auf keine Hieroglyphe mit einer Unterlänge. Bei den geringen Zeichenresten links vor der „Kinderhieroglyphe“ wage ich keine Lesung.

Da nun die Methode, den Textabstand zwischen der 2. und der 3. Zeile auf die 1. Zeile zu übertragen, Erfolg hatte, müßte man mit dem gleichen Abstand die Zeichengruppe $j w$ t3 der 
Zeile 4 (s. Abb. 4) bestimmen können. Dem ist aber nicht so. Schuld daran ist sicherlich z. T., daß wir nicht sicher sagen können, wie das Ende des Textes von Satet und Anuket aussah. Im hieroglyphischen „Buch vom Fayum“ bemerkt man $\langle 173,5 / 4,5\rangle$, daß die zweite Zeile (BFZeile 77-81) sehr gedrängt geschrieben und der Platz am Zeilenende immer dichter beschrieben wurde. Am Ende der Satet-Anuket-Zeile finden wir nun ein erstes $j w t 3$, das aber eigentlich viel zu nah an der Nennung von Satet und Anuket steht. Die hieratische Parallele Botti F (8) hilft uns nicht weiter, denn an der entscheidenden Stelle ist der Text nicht vollständig erhalten. Im nachfolgenden Text kommt $j w t 3$ als $r$ t3 noch zweimal vor, in den BF-Zeilen 84 und 87 . Aufgrund des anzunehmenden Textabstandes kommt für die Bestimmung der Zeile 4 eigentlich nur die BFZeile 84 in Frage, die BF-Zeile 87 ist zu weit von der Nennung von Satet und Anuket entfernt.

Unbefriedigend bleibt, daß der auf $j w t 3$ folgende Zeichenrest nicht zu den Hieroglyphen der BF-Zeile 84 passen will und daß auch das vorangehende hieratische Zeichen $(h n \text { ? })^{9}$ sich einer eindeutigen Bestimmung bzw. Zuordnung verweigert.

Bei der Bestimmung der 4. Zeile von pCarlsberg $310 \mathrm{kann}$ m. E. nur der Vergleich mit Botti F helfen. Hier beginnt der Satet-Anuket-Text in der 7. Zeile und endet genau eine Zeile tiefer. Dazwischen fehlt ein Textteil, der etwa so breit war wie das Papyrusfragment. Von dem Inhalt wissen wir einiges durch die hieroglyphische Textversion. Trotzdem scheinen mir noch an der Länge der Zeile ein paar Worte zu fehlen. Ich möchte deshalb vorschlagen, das Zeilenende so zu

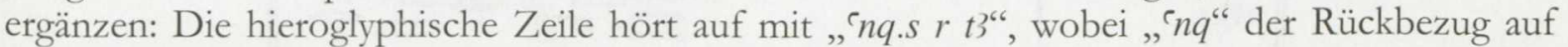
Anuket ist. Die Zeile 4 von pCarlsberg 310 fängt mit einem Zeichen an, das man evtl. als ,,$h^{\prime \prime}$ lesen kann. Stellt man diese Lesung neben „„nq-herbeiführen“, so könnte man hier ein Verbum der Bewegung, das auf , $h n^{\prime \prime}$ endet bzw. so determiniert wird, erwarten. „Sie (Anuket) führt die Flut herbei (nach B/H/A) und läßt sie gehen zum Land (nach pCarlsberg 310) von Mehu (nach Botti F)." Der kleine Strich am Ende der 4. Zeile von pCarlsberg 310, den ich in der Umschrift als Ideogrammstrich wiedergegeben habe, könnte sogar zu „mh“ gehören.

Nur wenn man die 4. Zeile des pCarlsberg 310 auf die genannte Weise identifiziert, läßt sich auch die 5. Zeile zuordnen (s. Abb. 5). Zu erkennen sind m. E. deutlich zwei Arme (Gardiner, EG, G 37) untereinander, was nur ein Rest der Schreibung für ḥn.t sein kann, wie sie im „Buch vom Fayum" gebräuchlich ist. Nun gibt es aber nach der BF-Zeile 84 für lange Zeit keine Nennung von hn.t. Wir finden den Ausdruck erst wieder in der BF-Zeile 195 und, gefolgt von einem $j$ wie auf unserem Fragment, sogar erst in der Zeile 491. Dabei könnte die Formulierung auf dem Papyrusfragment so ähnlich sein, wie in der BF-Zeile 491: ... hnn.t $r(=j w) \zeta$... Solch ein Text könnte auch durchaus in den Übergangsbereich vom 1. bis zum 3. Papyrusabschnitt, den Übergangsbereich vom „Hent zum See“ passen. M. E. gibt es nur eine Möglichkeit, die Zeilenreste des pCarlsberg 310 einzuordnen, wenn man nämlich unterstellt, daß die Präposition $r$ auf dem hieratischen Papyrus der Präposition $m$ im hieroglyphischen Text (BF-Zeile 84) entspricht.

\section{2. pBerlin 14.473b, Taf. 3}

Bei dem Papyrus handelt es sich um ein Fragment von 3,2 cm Breite und 6,8 $\mathrm{cm}$ Höhe. Die Zeilen sind liniert, der Abstand der Linien beträgt 1,0 cm. Es wurde schon einmal 1986 von Frau Kaplony-Heckel im Verzeichnis der orientalischen Handschriften in Deutschland, Band XIX, 3, aufgenommen, die aber die ganze 2. Zeile falsch gelesen hat und deshalb nicht in der Lage war, das Fragment zu identifizieren ${ }^{10}$. In der zweiten Zeile findet sich ein Rubrum, nämlich die Zahl 66, die Frau Kaplony als 120 liest, wobei mir allerdings kein Fall bekannt wäre, in dem man 120 mit 12 Zehnerzeichen geschrieben hätte.

\footnotetext{
9 Möller, Paläographie, Nr. 268, vgl. Botti, Taf. III, 7.

${ }^{10}$ Nach Frau Kaplony handelt es sich um einen Text ,wohl religiösen Inhalts ... (mit) Angabe der ,Ellen-Maße von $[\ldots]^{\text {cc }}$.
} 


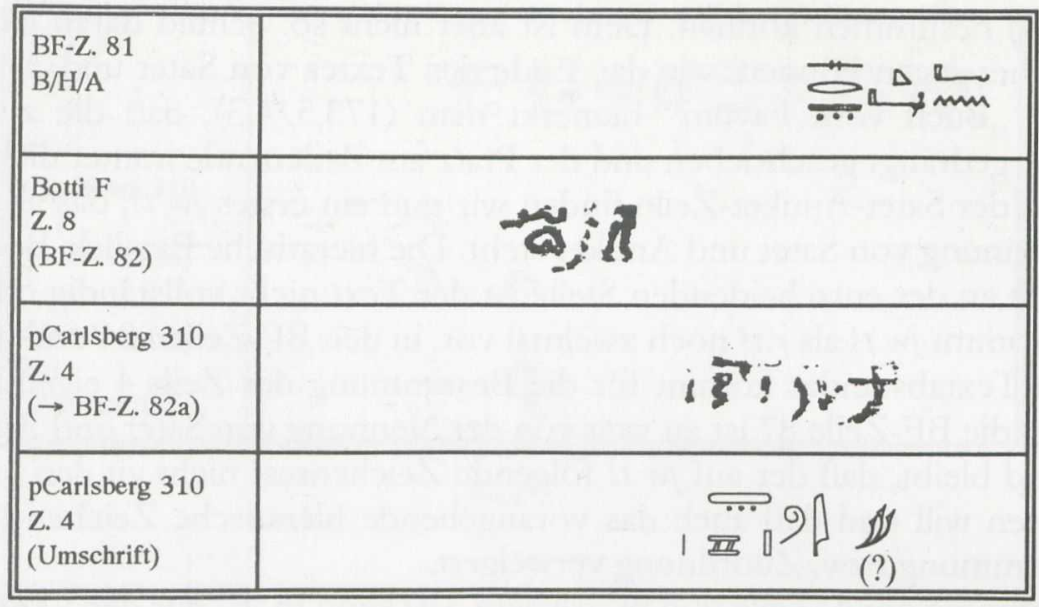

Abb. 4

\begin{tabular}{|c|c|}
\hline $\begin{array}{l}\text { BF-Z. } 84 \\
\text { B/H/A }\end{array}$ & 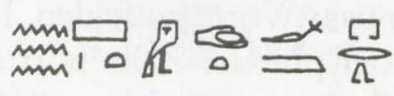 \\
\hline $\begin{array}{l}\text { pCarlsberg } 310 \\
\text { Z. } 5 \\
(\rightarrow \text { BF-Z. } 85 a)\end{array}$ & Pr \\
\hline $\begin{array}{l}\text { pCarlsberg } 310 \\
\text { Z. } 5 \\
\text { (Umschrift) }\end{array}$ & $\square 9 / \stackrel{\square}{\sim}$ \\
\hline
\end{tabular}

Abb. 5

Auf dem Papyrusfragment sind Reste von 7 Zeilen erhalten, von der letzten Zeile allerdings nur ein nicht identifizierbarer Rest. Bei den anderen Zeilen handelt es sich um Parallelen zu den BF-Zeilen 496 bis 517.

In der 1. Zeile (s. Abb. 6) läßt sich w3 $\underline{d}$ sicher erkennen, während die Zeichenreste links und rechts davon für eine Identifizierung nicht ausreichen. Da sich die nachfolgende Zeile genau bestimmen läßt, ist die 1. Zeile mit der BF-Zeile $496 \mathrm{zu}$ verbinden. Bei der 2. Zeile (s. Abb. 7) handelt es sich um die Berechnung der Gaue am Ende des 3. Papyrusabschnittes des „Buches vom Fayum“, BF-Zeile 501. Das erste Zeichen ist sicher als dmd (Möller, Paläographie, Nr. 421) zu erkennen, das von Frau Kaplony gelesene mh (Möller, Paläographie, Nr. 459) sieht ganz anders aus. Hinter dem Rubrum der Zahl 66 erkennt man noch die Reste von šm

Die dritte Zeile (s. Abb. 8) des Papyrusfragments ist parallel zu BF-Zeile 504: ... ntr.wt jm.f df $3 . w$... Wie in der hieroglyphischen Parallele, aber anders als in der Transliteration der Handschrift „Botti A“ steht zwischen jm.f und $\underline{d f} 3 . w$ kein $m$. Ich möchte deshalb annehmen, daß auch „Botti A, Taf. III, 9“ entsprechend zu lesen ist ${ }^{11}$.

Die Textzeile, die im hieroglyphischen „Buch vom Fayum“ den dritten Papyrusabschnitt und gleichzeitig die erste Hälfte des Buches abschließt, reicht von der 3. Zeile (s. Abb. 9) des Berliner

${ }^{11}$ Entgegen meinem Vorschlag im „Buch vom Fayum“, S. 189 ist wohl aufgrund der Parallele des Berliner Papyrusfragments das letzte Zeichen der Gruppe kein $w$, sondern ein $f$. 


\begin{tabular}{||l|l||}
\hline $\begin{array}{l}\text { BF-Z. 496 } \\
\text { B/H/A }\end{array}$ & \\
\hline $\begin{array}{l}\text { Botti A } \\
\text { III, Z. 7 } \\
\text { (BF-Z. 497) }\end{array}$ & \\
\hline $\begin{array}{l}\text { pBerlin 14473b } \\
(\rightarrow \text { BF-Z. 497a) }\end{array}$ & \\
\hline $\begin{array}{l}\text { pBerlin 14473b } \\
\text { Z. 1 } \\
(\text { Umschrift })\end{array}$ & \\
\hline
\end{tabular}

Abb. 6

\begin{tabular}{|c|c|}
\hline $\begin{array}{l}\text { BF-Z. } 501 \\
\text { B/H/A }\end{array}$ & 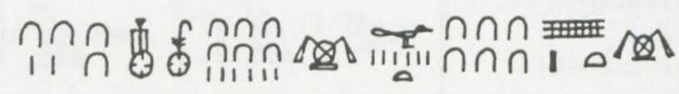 \\
\hline $\begin{array}{l}\text { Botti A } \\
\text { III, Z. } 8 \\
\text { (BF-Z. 502) }\end{array}$ & (1) \\
\hline $\begin{array}{l}\text { pBerlin 14473b } \\
\text { Z. } 2 \\
(\rightarrow \text { BF-Z. 504a) }\end{array}$ & 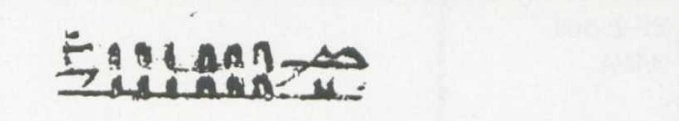 \\
\hline $\begin{array}{l}\text { pBerlin 14473b } \\
\text { Z. } 2 \\
\text { (Umschrift) }\end{array}$ & 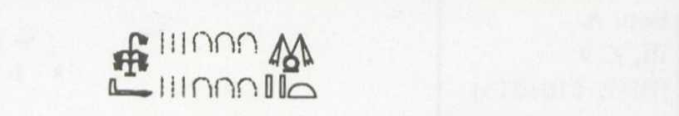 \\
\hline
\end{tabular}

Abb. 7

Papyrusfragments bis in die 4. Zeile (s. Abb. 10). Das ist auffällig, weil der Abstand zwischen den beiden Textstellen (Bf-Zeile 506 und 509) eigentlich sehr kurz ist. Die abschließenden Zeichen (mjt.t jrj) sind klar zu lesen. Sie bestätigen die Textwiedergabe des hieroglyphischen „Buches vom Fayum“ und erlauben wiederum eine Korrektur der Transliteration von Botti A, wo das letzte Wort sicher auch jrj zu lesen ist. Das nachfolgende Rubrum s.t tn ist die Einleitung zum 4. Papyrusabschnitt des „Buches vom Fayum“, der den Ort Schedet behandelt, und zu diesem Ort gehören auch die beiden letzten Zeilen ... mh.t ntr nt ... (Bf-Zeile 515) (s. Abb. 11) und ... hnww $n$ ḥr.wt ... (Bf-Zeile 517) (s. Abb. 12) ${ }^{12}$.

\section{3. pCarlsberg 311 (ehem. ICPCP 10.776), Taf. III}

Bei dem Papyrus handelt es sich um ein Fragment von 5,9 cm Breite und 19,5 cm Höhe, das recto und verso beschriftet ist. Recto erkennt man die Reste von 18 hieratischen Zeilen aus dem „Buch vom Fayum“, verso 21 Zeilen eines demotisch beschrifteten Papyrus, den man aber

${ }^{12}$ Das letzte Zeichen ist bei Kaplony falsch wiedergegeben. 


\begin{tabular}{|c|c|}
\hline $\begin{array}{l}\text { BF-Z. } 506 \\
\text { B/H/A }\end{array}$ & 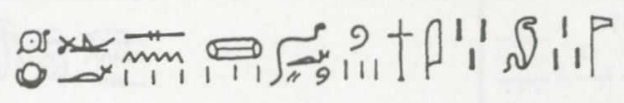 \\
\hline $\begin{array}{l}\text { Botti A } \\
\text { III, Z. } 9 \\
\text { (BF-Z. 502) }\end{array}$ & 203 \\
\hline $\begin{array}{l}\text { pBerlin } 14473 b \\
\text { Z. } 3 \\
(\rightarrow \text { BF-Z. 507a) }\end{array}$ & \\
\hline $\begin{array}{l}\text { pBerlin 14473b } \\
\text { Z. } 3 \\
\text { (Umschrift) }\end{array}$ & 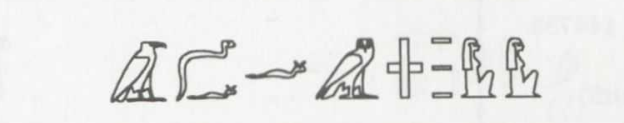 \\
\hline $\begin{array}{l}\text { pCarlsberg } 311 \\
\text { Z. } 2 \\
(\rightarrow \text { BF-Z. 507b) }\end{array}$ & \\
\hline $\begin{array}{l}\text { pCarlsberg } 311 \\
\text { Z. } 2 \\
\text { (Umschrift) }\end{array}$ & 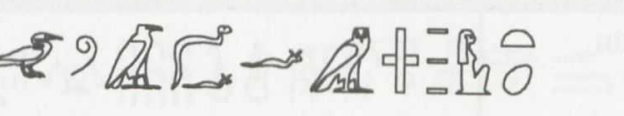 \\
\hline
\end{tabular}

Abb. 8

\begin{tabular}{|l|c||}
\hline $\begin{array}{l}\text { BF-Z. 509 } \\
\text { B/H/A }\end{array}$ & \\
\hline $\begin{array}{l}\text { Botti A } \\
\text { III, Z. 9 } \\
\text { (BF-Z. 510+513) }\end{array}$ & \\
\hline $\begin{array}{l}\text { Botti C } \\
\text { Z. . } \\
(\text { BF-Z. 511) }\end{array}$ & \\
\hline $\begin{array}{l}\text { pBerlin 14473b } \\
\text { Z. 4 } \\
(\rightarrow \text { BF-Z.511a) }\end{array}$ & \\
\hline $\begin{array}{l}\text { pBerlin 14473b } \\
\text { Z. 4 } \\
(\text { Umschrift) }\end{array}$ & 0 \\
\hline
\end{tabular}

Abb. 9

zum Beschriften so gedreht hat, daß die Oberkante des hieratisch beschrifteten Papyrus zur Unterkante des demotischen Papyrus wurde. Damit gleicht er dem oben besprochenen pCarlsberg 310, und man kann nicht ausschließen, daß die beiden Papyri, wenn auch weit voneinander entfernt, von der gleichen Papyrusrolle stammen. Auf der Vorderseite des Papyrus läßt sich Linierung feststellen. Unter der untersten Zeile ist ein etwa 3,5 cm hoher Rand. Wie schon z. T. pBerlin 14.473b gehört auch pCarlsberg 311 zum 4. Papyrusabschnitt des Buches vom Fayum und zwar zu dem Abschnitt, der ansonsten nur sehr schlecht erhalten ist. Der Papyrus umfaßt die BF-Zeilen 506 bis 629. Die Reste der obersten Zeile des Fragments reichen für eine Identifizierung nicht aus. Zu erkennen sind hier lediglich zwei Unterlängen. 


\begin{tabular}{|l|l|||}
\hline $\begin{array}{l}\text { Botti A } \\
\text { III Z. } 10\end{array}$ & (BF-Z. 513-515)
\end{tabular}

Abb. 10

\begin{tabular}{||l|l||}
\hline $\begin{array}{l}\text { Botti A } \\
\text { III, Z. 11 } \\
\text { (BF-Z. 517) }\end{array}$ & \\
\hline $\begin{array}{l}\text { pBerlin 14473b } \\
(\rightarrow \text { BF-Z.517a) }\end{array}$ & \\
\hline $\begin{array}{l}\text { pBerlin 14473b } \\
\text { Z. } 6 \\
(\text { Umschrift })\end{array}$ & \\
\hline
\end{tabular}

Abb. 11

\begin{tabular}{||l|l||}
\hline $\begin{array}{l}\text { Botti A } \\
\text { III, Z. } 11 \\
(\text { BF-Z. 520-521) }\end{array}$ & \\
\hline $\begin{array}{l}\text { pCarlsberg } 311 \\
\text { Z. } 4 \\
(\rightarrow \text { BF-Z. 521a) }\end{array}$ & \\
\hline $\begin{array}{l}\text { pCarlsberg } 311 \\
\text { Z. } 4 \\
(\text { Umschrift })\end{array}$ & \\
\hline
\end{tabular}

Abb. 12 


\begin{tabular}{|c|c|}
\hline $\begin{array}{l}\text { Botti A } \\
\text { III, Z. } 12 \\
\text { (BF-Z. 526) }\end{array}$ & 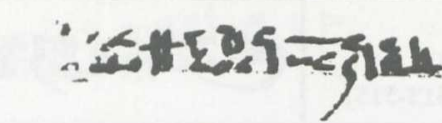 \\
\hline $\begin{array}{l}\text { pCarlsberg } 311 \\
\text { Z. } 5 \\
(\rightarrow \text { BF-Z. 526a) }\end{array}$ & 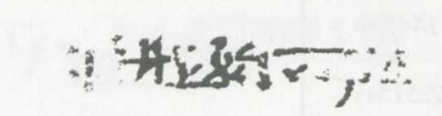 \\
\hline $\begin{array}{l}\text { pCarlsberg } 311 \\
\text { Z. } 5 \\
\text { (Umschrift) }\end{array}$ & $\begin{array}{l}08 R 08 \mathrm{~mm} \\
\triangle 1100801090\end{array}$ \\
\hline
\end{tabular}

Abb. 13

\begin{tabular}{|c|c|}
\hline $\begin{array}{l}\text { Botti A } \\
\text { III, Z. } 13-14 \\
\text { (BF-Z. } 532+535)\end{array}$ & tofigk IIt!. \\
\hline $\begin{array}{l}\text { pCarlsberg } 311 \\
\text { Z. } 6 \\
(\rightarrow \text { BF-Z. 532a) }\end{array}$ & Tinifind \\
\hline $\begin{array}{l}\text { pCarlsberg } 311 \\
\text { Z. } 6 \\
\text { (Umschrift) }\end{array}$ & 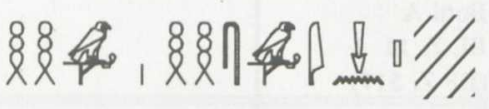 \\
\hline
\end{tabular}

Abb. 14

Die 2. Zeile (s. Abb. 8) des Fragments stellt eine Parallele zur 3. Zeile des oben besprochenen Berliner Fragments dar, entspricht also der BF-Zeile 506. Die Zeilen 3 bis 5 (s. Abb. 10, 12 u. 13) bieten keine besonderen Probleme. Sie sind Parallelen zu den BF-Zeilen 513/515, 520/521 und 526.

Der Anfang der 6. Zeile (s. Abb. 14) macht Schwierigkeiten, weil die einzige Parallele, Botti A, III, 13, an dieser Stelle eine Lücke hat. Davor finden wir eine Aufzählung von Göttinnen, deren Bedeutung aber auch unklar ist: „Taschit. Repit. ,Die im Gebiete ihres (Fremd)landes ist. ‘,Die im Besitze ihres ..." Der letzte Name wird durch die Parallele des pCarlsberg 311 etwas klarer: „Die im Besitze des ... ihres Bruders ist.“ Der Ausdruck „... ihres Bruders“ deutet dabei darauf hin, daß es sich um eine Isis-Osiris-Beziehung handelt. Das fehlende Wort sollte also etwas aus dem Bereich des Todes oder Begräbnisses sein. Links neben dem unleserlichen Zeichen scheint ein Ideogrammstrich zu sein, was vermuten läßt, daß das fragliche Zeichen ein Ideogramm ist. Das Zeichen selbst ist geschwungen und zieht am Zeilenboden stark ein. Man würde an einen Vogelrücken denken, dagegen spricht aber, daß die geschwungene Linie die ganze Zeilenhöhe einnimmt. Evtl. könnte man an š̌d-Binden denken (vgl. Möller, Paläographie, Nr. 522). Die Lesung des Urgötterpaares Huh und Hauhet am Zeilenende ist unproblematisch.

Auch die 7. Zeile (s. Abb. 15) des Papyrusfragments hat keine eindeutige Parallele. Die BFZeilen 541 und 545 sind im Hieratischen und im Hieroglyphischen nicht vollständig erhalten. Erfreulicherweise setzt unser Text genau dort ein, wo die Version Botti A (III, 15) endet, bei $m 3 w j-$ sich erneuern. Schwieriger zu bestimmen ist das nächste Zeichen, das in der Handschrift Botti A zum Teil ebenfalls erhalten ist, von Botti bei der Umschrift des Textes aber unberücksichtigt gelassen wurde. Ich halte es für eine hieratische Wiedergabe des Zeichens hn (V36A) mit der Bedeutung „Auftrag“, kann aber keine hieratische Form dieses Zeichens anführen. Der Text über die Urgötter heißt nach dieser Ergänzung für die BF-Zeile 541 bis 545: „(Thre) Leiber sind 


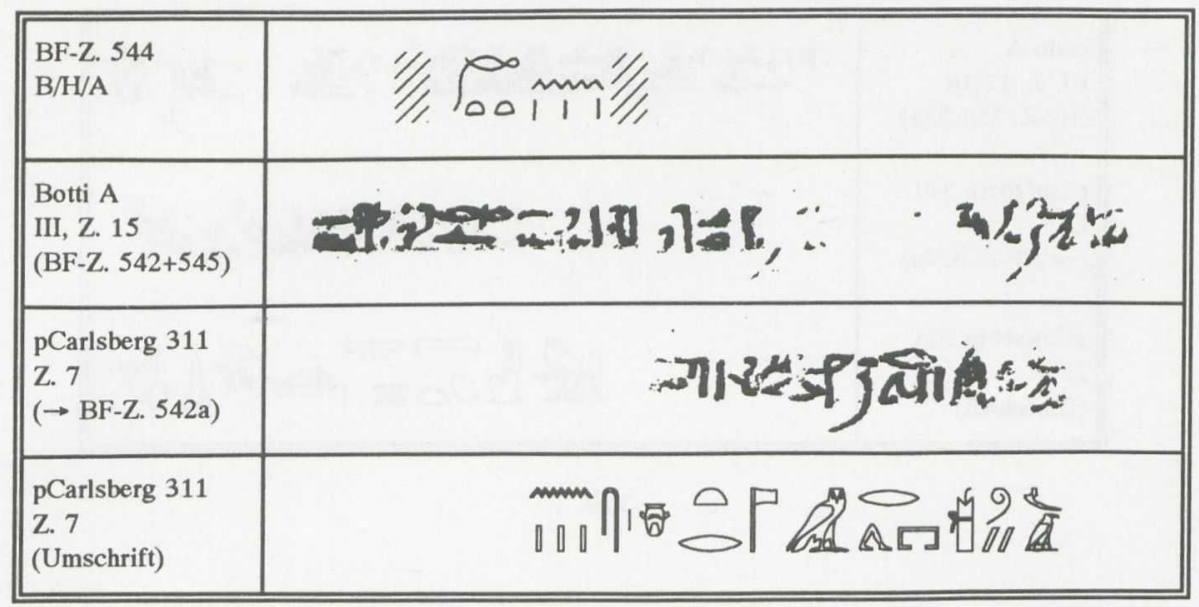

Abb. 15

\begin{tabular}{|c|c|}
\hline $\begin{array}{l}\text { Botti A } \\
\text { III, Z. } 16 \\
\text { (BF-Z. } 550)\end{array}$ & 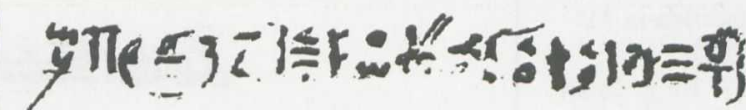 \\
\hline $\begin{array}{l}\text { pCarlsberg } 311 \\
\text { Z. } 8 \\
(\rightarrow \text { BF-Z. 550a) }\end{array}$ & 3 넌 \\
\hline $\begin{array}{l}\text { pCarlsberg } 311 \\
\text { Z. } 8 \\
\text { (Umschrift) }\end{array}$ & 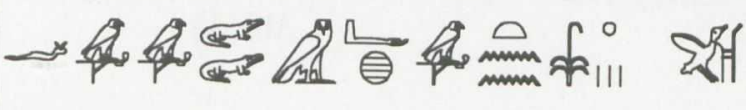 \\
\hline
\end{tabular}

Abb. 16

zufriedengestellt im Wadj-wer beim Erneuern des Auftrages, der aus ihren Herzen kommt. Sie schützen Re im Inneren der Kapelle“. Gemeint ist dabei wohl die Vorstellung vom Fayum-See, den die Urgötter gegründet haben und in dem sie leben (BF-Zeile 1241ff.). Der Auftrag, der immer wieder von neuem aus ihrem Herzen kommt, ist die Nilflut, denn das Fayum ist der „Ort, an dem die Achtheit die Flut entstehen läßt“ (BF-Zeile 129).

Auch aufgrund der 8. Zeile (s. Abb. 16) des Papyrusfragments müssen Korrekturen an der bisherigen Lesung und Übersetzung angebracht werden: Frisch ist das Herz der Weißen, „die erschaffen hat das ... des Königs und seine(r) Achemu“. Es ist höchst bedauerlich, daß das Wort hinter qm3, das auch im Text Botti A (II, 16) so deutlich zu sein scheint und auch in unserem Text immerhin erkennbar ist, sich nicht identifizieren läßt. Man kann deshalb auch nicht sagen, ob von diesem Wort auch Achemu abhängig ist, oder ob die Achemu parallel zu den ... des Königs geschaffen wurden. Immerhin kann man das Wort Achemu deutlich erkennen und damit die Transliteration Bottis verbessern. Eine Besonderheit der Zeile ist ohne Zweifel auch die reizvolle Schreibung der beiden Krokodile übereinander als Determinativ zu Achemu. Sie erinnert doch sehr an die Darstellung der beiden Krokodile im illustrierten „Buch vom Fayum“613.

Die 9. Zeile (s. Abb. 17) ist parallel zu BF-Zeile 554/556: ,... die (p3w.tj)-Urgottheit (shm.tj?). Durchfahren das Land der Nut, die Wege ...". Leider wird nicht gesagt, wer diese Fahrt macht, und auch sonst bleibt der Text dunkel.

13 „Buch vom Fayum“, S. 94, Abb. 33 und $<424 / 6>$. 


\begin{tabular}{|l|r|}
\hline $\begin{array}{l}\text { Botti A } \\
\text { III, Z. 17-18 }\end{array}$ & (BF-Z. 554-556)
\end{tabular}

Abb. 17

\begin{tabular}{|l|l|}
\hline $\begin{array}{l}\text { Botti A } \\
\text { III, Z. 19 } \\
\text { (BF-Z. 560) }\end{array}$ & \\
\hline $\begin{array}{l}\text { pCarlsberg 311 } \\
\begin{array}{l}\text { (. } 10 \\
(\rightarrow \text { BF-Z. 560a) }\end{array}\end{array}$ \\
\hline $\begin{array}{l}\text { pCarlsberg 311 } \\
\text { Z. 10 } \\
(\text { Umschrift) }\end{array}$
\end{tabular}

Abb. 18

\begin{tabular}{|c|c|}
\hline $\begin{array}{l}\text { Botti A } \\
\text { IV, Z. } 1 \\
\text { (BF-Z. 563) }\end{array}$ & $\sqrt{1}$ \\
\hline $\begin{array}{l}\text { pCarlsberg } 311 \\
\text { Z. } 11 \\
(\rightarrow \text { BF-Z. 563a) }\end{array}$ & $4 \%$ \\
\hline $\begin{array}{l}\text { pCarlsberg } 311 \\
\text { Z. } 11 \\
\text { (Umschrift) }\end{array}$ & 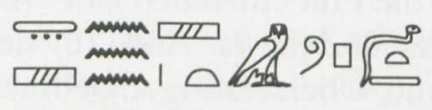 \\
\hline
\end{tabular}

Abb. 19

Die nächste Zeile (Z. 10) (s. Abb. 18) füllt zwar wieder eine Lücke im Papyrus Botti A (III, 19), aber gerade dort findet sich wieder ein nicht lesbares hieratisches Zeichen. Botti A notiert als letzte gut lesbare Gruppe vor der Lücke „Wüstenbewohner (h3s.tjw)“. Die nächste Gruppe transliteriert Botti als rmtw (Menschen), obwohl man das $r$ nur bei bestem Wohlwollen als solches ansehen kann. Am ehesten würde ich es hm (Möller, Paläographie III, Nr. 98) lesen. Dieses Zeichen könnte auch hinter dem bei Botti A stecken, wenn man einmal die Version des pRhind dazu vergleicht. Leider ist es mir aber auch nicht gelungen, die gemeinte Menschengruppe zu identifizieren. Ich vermute, daß es sich um Leute handelt, die parallel zu den Wüstenbewohnern genannt werden können, etwa Oasenbewohner. Dabei werde ich sicher von einer weiteren Vermutung beeinflußt: Hinter dieser Gruppe folgt wn-hntjw, und ich glaube, dahinter noch die Reste eines Mannes mit Pluralstrichen darunter erkennen zu können. Diese Gruppe könnte man evtl. als Reisende oder Nomaden bezeichnen. Wenn dies zutreffen sollte, müßte die 


\begin{tabular}{|c|c|}
\hline $\begin{array}{l}\text { Botti A } \\
\text { IV, Z. } 2 \\
\text { (BF-Z. 571-573) }\end{array}$ & $471-35116$ \\
\hline $\begin{array}{l}\text { pCarlsberg } 311 \\
\text { Z. } 12 \\
(\rightarrow \text { BF-Z. 571a) }\end{array}$ & 7 flil \\
\hline $\begin{array}{l}\text { pCarlsberg } 311 \\
\text { Z. } 12 \\
\text { (Umschrift) }\end{array}$ & 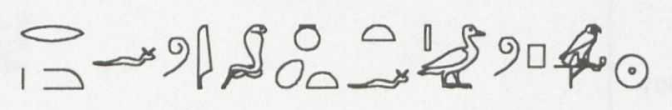 \\
\hline
\end{tabular}

Abb. 20

\begin{tabular}{|c|c|}
\hline $\begin{array}{l}\text { Botti A } \\
\text { IV, Z. } 3 \\
\text { (BF-Z. 580-581) }\end{array}$ & 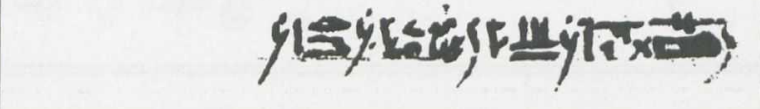 \\
\hline $\begin{array}{l}\text { pCarlsberg } 311 \\
\text { Z. } 13 \\
(\rightarrow \text { BF-Z. 580a) }\end{array}$ & 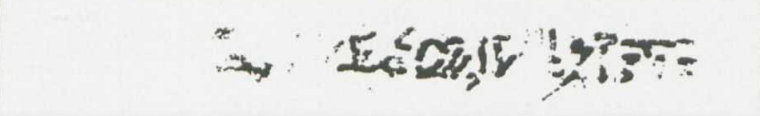 \\
\hline $\begin{array}{l}\text { pCarlsberg } 311 \\
\text { Z. } 13 \\
\text { (Umschrift) }\end{array}$ & 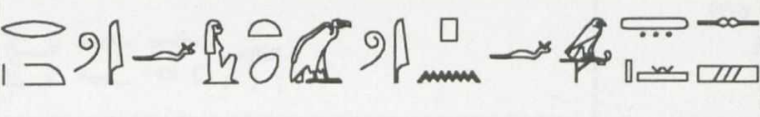 \\
\hline
\end{tabular}

Abb. 21

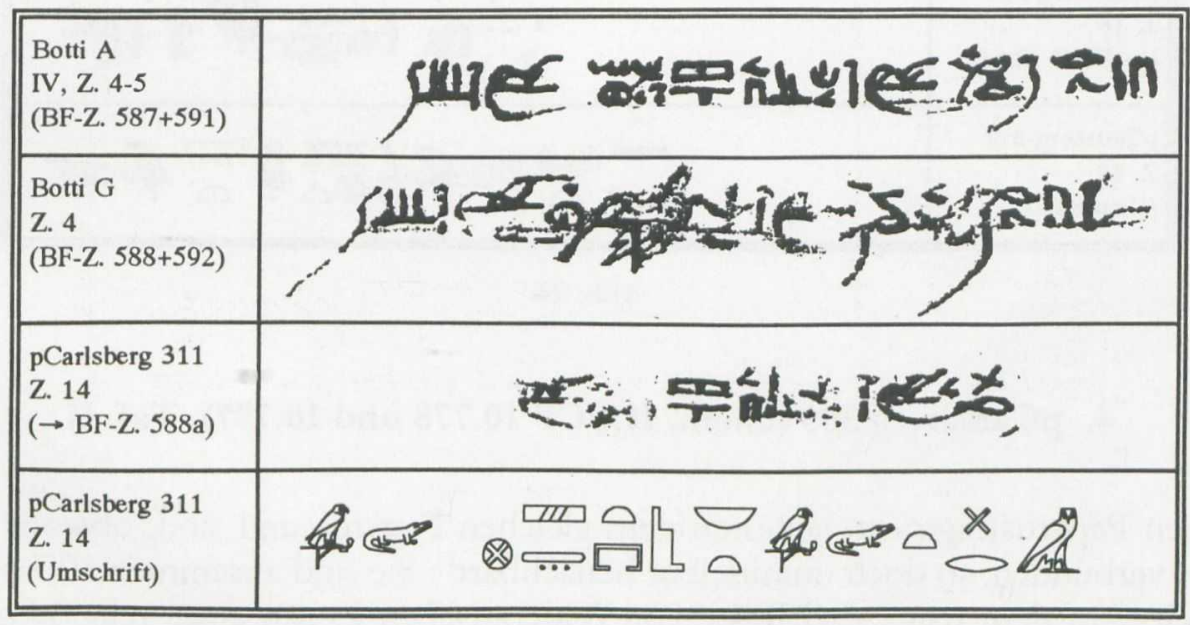

Abb. 22

unleserliche Gruppe am Anfang unserer Zeile inhaltlich zwischen Wüstenbewohnern und Reisenden/Nomaden angesiedelt werden.

Der Rest des Papyrusfragments ist unproblematisch (s. Abb. 19-26). Es gibt kaum eine Abweichung vom bisher bekannten Text, außer daß am Ende der 15. Zeile (BF-Zeile 597) unser Papyrusfragment eine ganze Passage ausläßt, in der es heißt, daß Sobek vom 2. o.äg. Gau die Feinde seines Vaters Re vertrieben hat. 


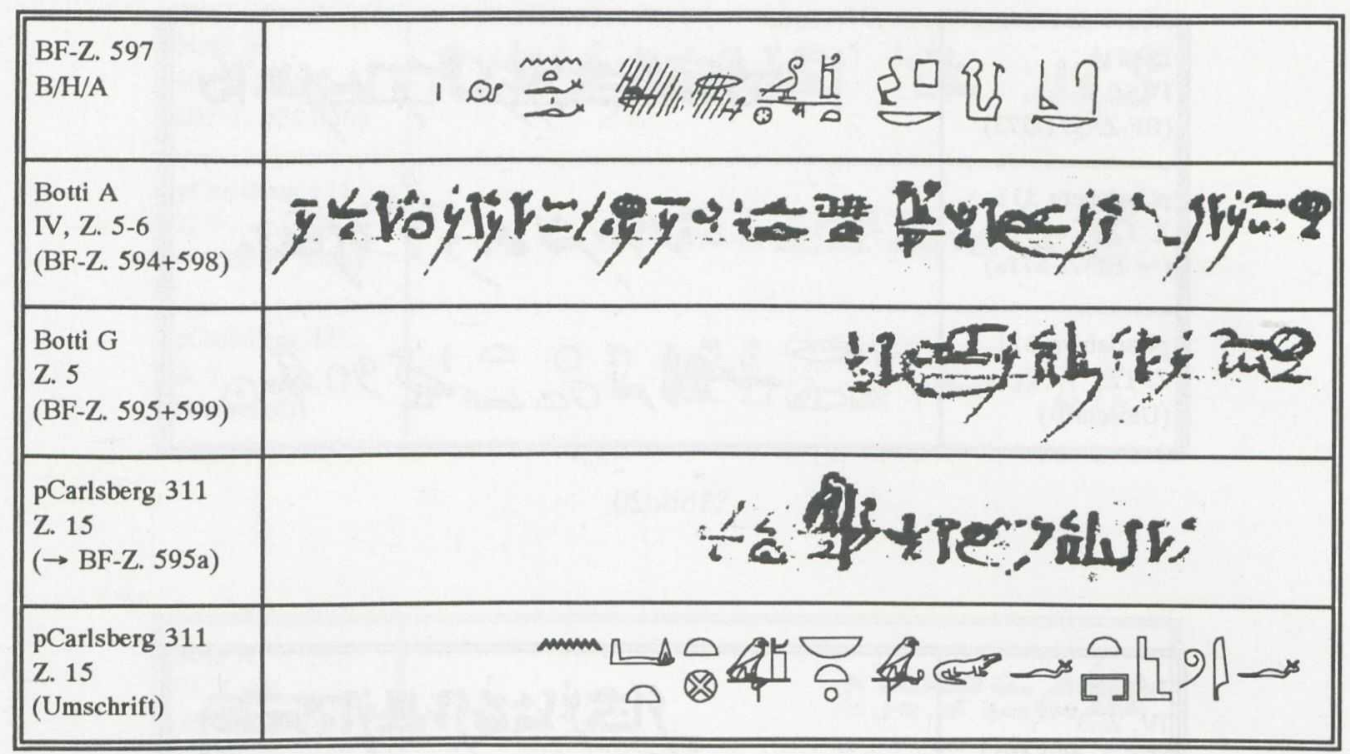

Abb. 23

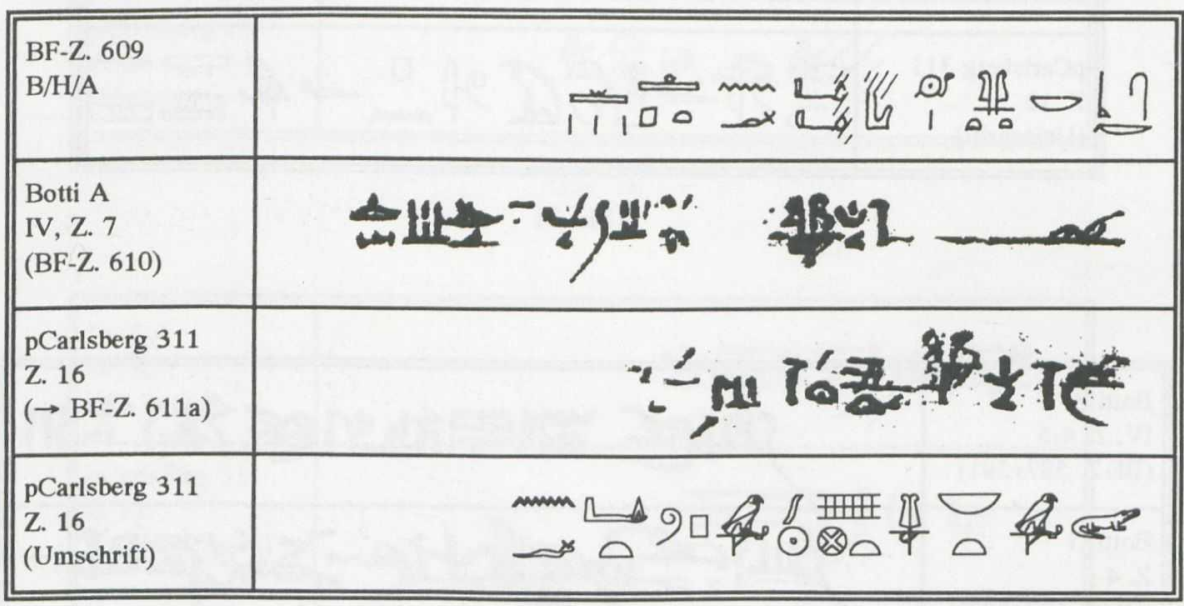

Abb. 24

4. pCarlsberg 309 (ehem. ICPCP 10.778 und 10.777), Taf. II

Die beiden Papyrusfragmente gehören zum gleichen Papyrus und sind, obwohl nicht direkt miteinander verbunden, so doch unmittelbar benachbart ${ }^{14}$. Sie sind zusammen $10,7 \mathrm{~cm}$ hoch und $11,4 \mathrm{~cm}$ breit. Auf dem recto sind Reste von 6 hieratischen Zeilen eines religiösen Textes mit Nennungen von Göttinnen zu erkennen. Das verso ist liniert. Hier sieht man Reste von 10 hieratischen Zeilen des „Buches vom Fayum“. Der untere Rand ist verso $6,2 \mathrm{~cm}$, recto 2,6 cm hoch.

Die oberste Zeile des Doppelfragments ist nur noch in äußerst geringen Resten erhalten. Sicherlich gehörte sie wie auch die restlichen Zeilen zum 5. Papyrusabschnitt (Ra-sehui) des „Buches vom Fayum“. Genauer gesagt finden wir in den lesbaren Zeilen Parallelen zu den BF-

\footnotetext{
${ }^{14}$ Ich danke Herrn Ryholt dafür, daß er meinen Rekonstruktionsvorschlag am Original überprüft hat.
} 


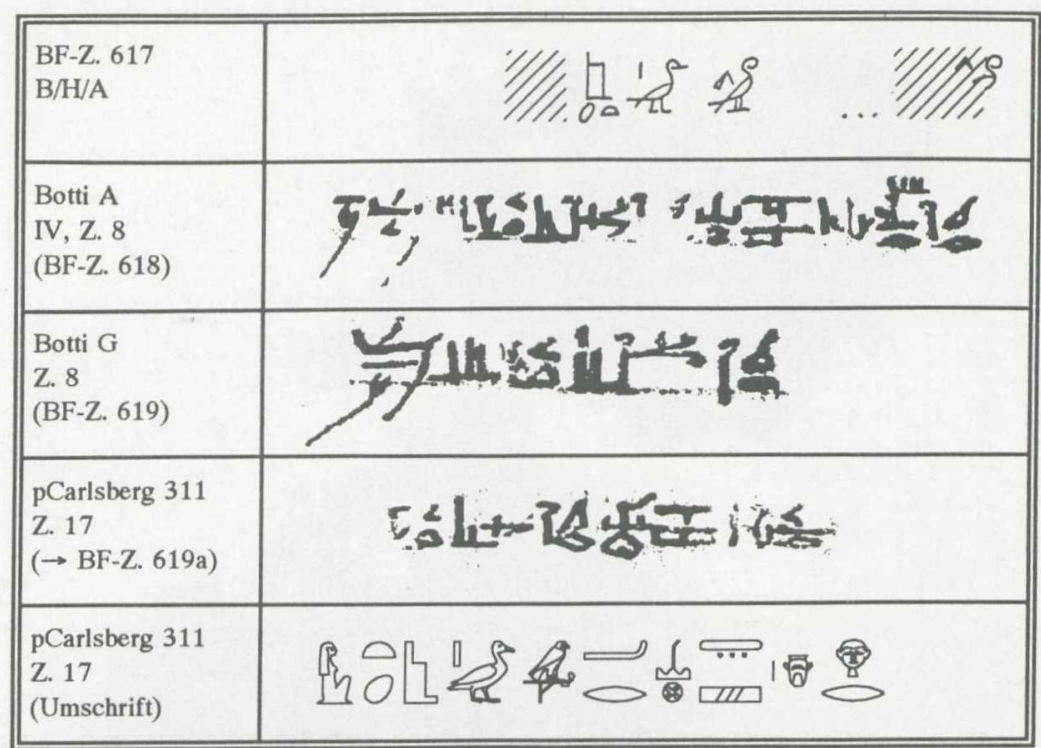

Abb. 25

\begin{tabular}{|c|c|}
\hline $\begin{array}{l}\text { BF-Z: } 629 \\
\text { B/H/A }\end{array}$ & $\left.1 / 1 /, \frac{s \AA}{30} 0\right\rfloor ?$ \\
\hline $\begin{array}{l}\text { Botti A } \\
\text { IV, Z. } 9 \\
\text { (BF-Z. 630) }\end{array}$ & 6 \\
\hline $\begin{array}{l}\text { Botti G } \\
\text { Z. } 9 \\
\text { (BF-Z. 631) }\end{array}$ & $A 2$ \\
\hline $\begin{array}{l}\text { pCarlsberg } 311 \\
\text { Z. } 18 \\
(\rightarrow \text { BF-Z. 631a) }\end{array}$ & $\because$ MT Plf \\
\hline $\begin{array}{l}\text { pCarlsberg } 311 \\
\text { Z. } 18 \\
\text { (Umschrift) }\end{array}$ & 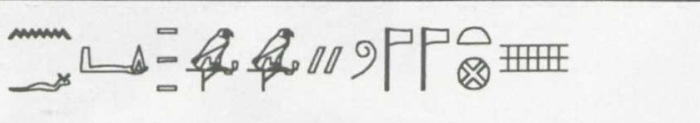 \\
\hline
\end{tabular}

Abb. 26

\begin{tabular}{|c|c|c|}
\hline $\begin{array}{l}\text { Botti A } \\
\text { VI, Z. } 11 \\
\text { (BF-Z. } 950+954 \text { ) }\end{array}$ & 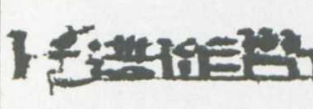 & 101 \\
\hline $\begin{array}{l}\text { pCarlsberg } 309 \\
\text { Z. } 2 \\
(\rightarrow \text { BF-Z. 950a) }\end{array}$ & Zija & 12.7961 \\
\hline $\begin{array}{l}\text { pCarlsberg } 309 \\
\text { Z. } 2 \\
\text { (Umschrift) }\end{array}$ & 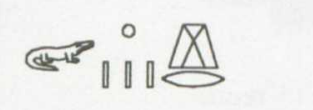 & 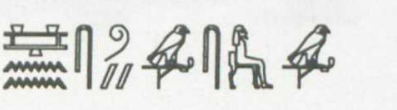 \\
\hline
\end{tabular}


TAFEL II

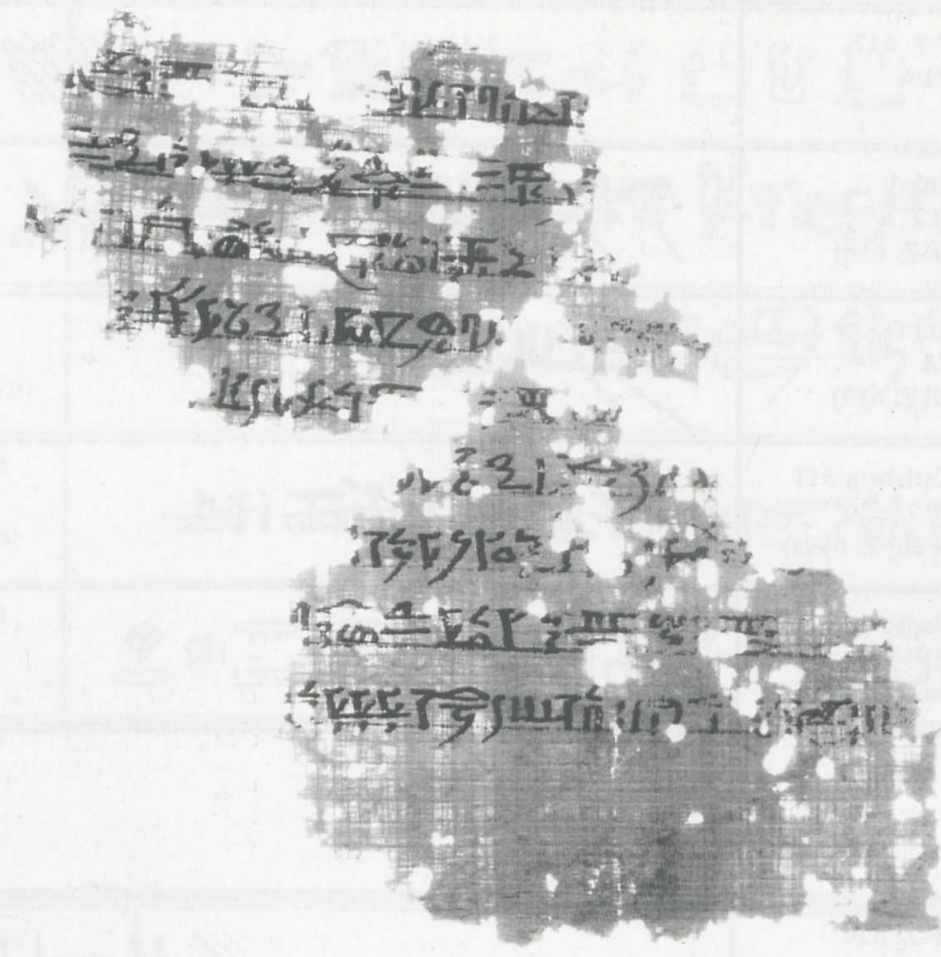

verso

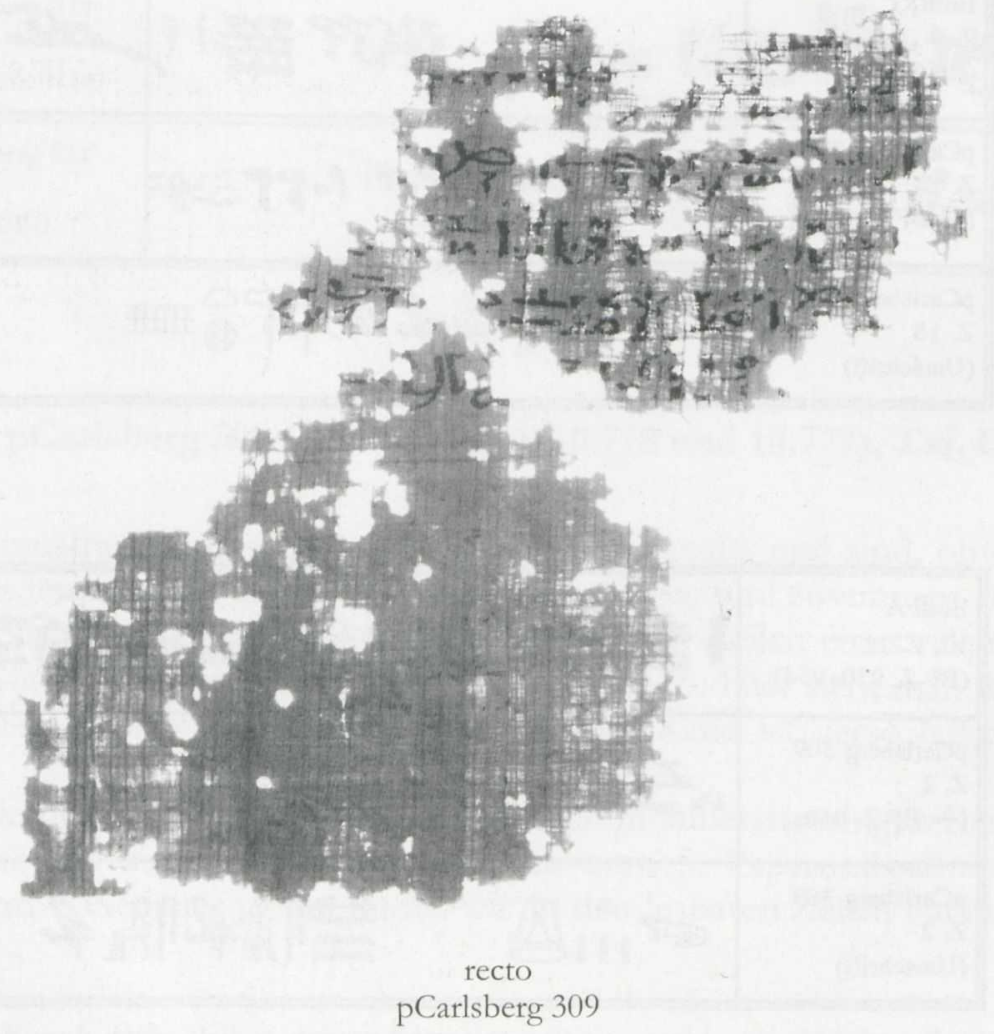


Zeilen 947 bis 1010 mit der Beschreibung des Lebenshauses. Die zweite Zeile (s. Abb. 27) setzt unmittelbar hinter der Textstelle ein, an der im Papyrus Botti A das vignettenartige Determinativ zu $s ? h$ (Mumie) steht. Das Götterdeterminativ dahinter ist zwar zu sehen und auch ein geringer Rest des voranstehenden Zeichens, für eine Bestimmung des Zeichens reicht dies aber nicht aus, besonders da man mit einer ungewöhnlichen Hieroglyphe rechnen muß. Der Rest der Zeile bietet keine Überraschungen und ist trotz der Lücken unter Zuhilfenahme der Parallele Botti A (VI, 11) leicht zu lesen. Am Zeilenende ist gerade noch der Anfang des Götternamens „SobekGeb“ zu erkennen, mit dem die Mumie des Lebenshauses von Ra-sehui gleichgesetzt wird.

In den nachfolgenden Zeilen finden wir Teile aus den Aufzählungen der Wissensbereiche ( $r h$ jh.t) des Lebenshauses, in Z. 3 (s. Abb. 28) Pflanzen, in Z. 4 (s. Abb. 29) (Götter und Göttin)nen der beiden Länder und der Neun-Bogen-Völker. Die Z. 4 leitet dann über zum Wissensbereich von Himmel, Erde und Dat, also zu den Geowissenschaften.

Die Z. 5 (s. Abb. 30) beginnt mit der Beschreibung der „Breiten Halle der Götter“, während die Z. 6 (s. Abb. 31) dann schon mitten in der Beschreibung der „Breiten Halle der Dinge" einsetzt. Es wird hier wohl der „Bedarf $(\underline{h r} . t)^{“}$ eines Mannes genannt und dieser Ausdruck mit "Ausstattung $(h n)$ “ interpretiert.

\begin{tabular}{|c|c|}
\hline $\begin{array}{l}\text { BF-Z.961 } \\
\text { B/H/A }\end{array}$ & 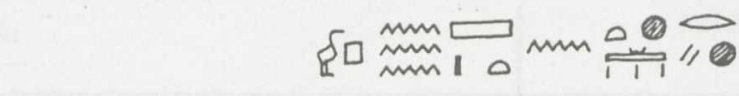 \\
\hline $\begin{array}{l}\text { BF-Z. } 962 \\
\text { B/H/A }\end{array}$ & 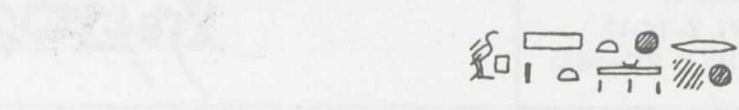 \\
\hline $\begin{array}{l}\text { Botti A } \\
\text { VI, Z. } 12 \\
\text { (BF-Z. } 963 \text { ) }\end{array}$ & 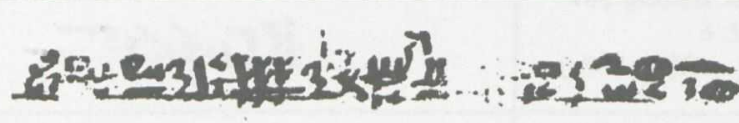 \\
\hline $\begin{array}{l}\text { pCarlsberg } 309 \\
\text { Z. } 3 \\
(\rightarrow \text { BF-Z. 963a) }\end{array}$ & 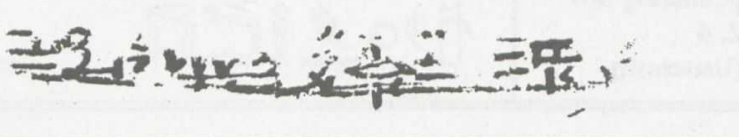 \\
\hline $\begin{array}{l}\text { pCarlsberg } 309 \\
\text { Z. } 3 \\
\text { (Umschrift) }\end{array}$ & 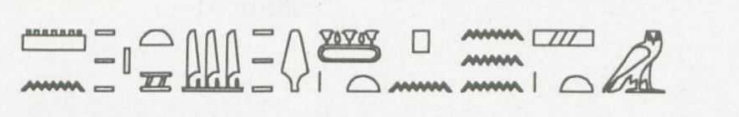 \\
\hline
\end{tabular}

Abb. 28

\begin{tabular}{|c|c|}
\hline $\begin{array}{l}\text { BF-Z. } 968 \\
\text { B/H/A }\end{array}$ & 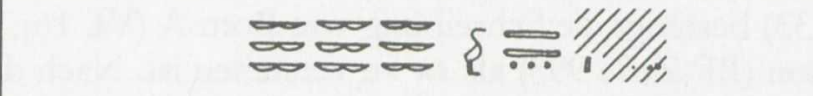 \\
\hline $\begin{array}{l}\text { Botti A } \\
\text { VI, Z. } 13 \\
\text { (BF-Z. } 969+972)\end{array}$ & 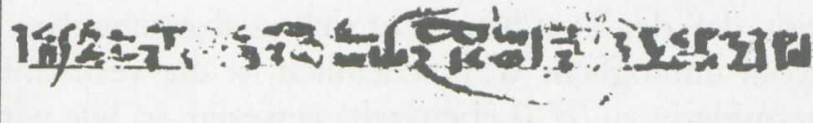 \\
\hline $\begin{array}{l}\text { pCarlsberg } 309 \\
\text { Z. } 4 \\
(\rightarrow \text { BF-Z. 969a) }\end{array}$ & 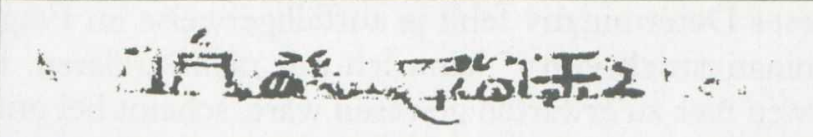 \\
\hline $\begin{array}{l}\text { pCarlsberg } 309 \\
\text { Z. } 4 \\
\text { (Umschrift) }\end{array}$ & 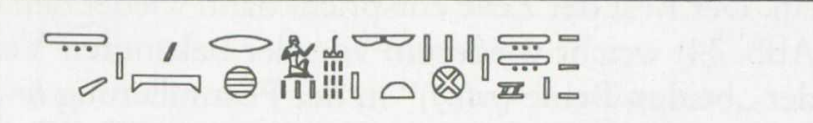 \\
\hline
\end{tabular}

Abb. 29 


\begin{tabular}{|c|c|}
\hline $\begin{array}{l}\text { BF-Z. } 977 \\
\text { B/H/A }\end{array}$ & 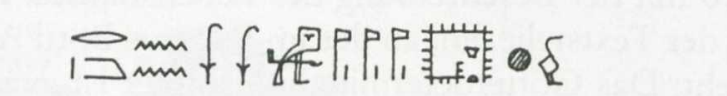 \\
\hline $\begin{array}{l}\text { Botti A } \\
\text { VI, Z. } 14 \\
\text { (BF-Z. 978) }\end{array}$ & ins \\
\hline $\begin{array}{l}\text { pCarlsberg } 309 \\
\text { Z. } 5 \\
(\rightarrow \text { BF-Z. 978a) }\end{array}$ & $F 52 \times 3 \times 1,5$ \\
\hline $\begin{array}{l}\text { pCarlsberg } 309 \\
\text { Z. } 5 \\
\text { (Umschrift) }\end{array}$ & 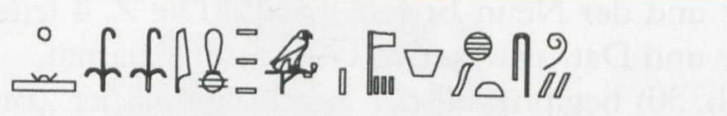 \\
\hline
\end{tabular}

Abb. 30

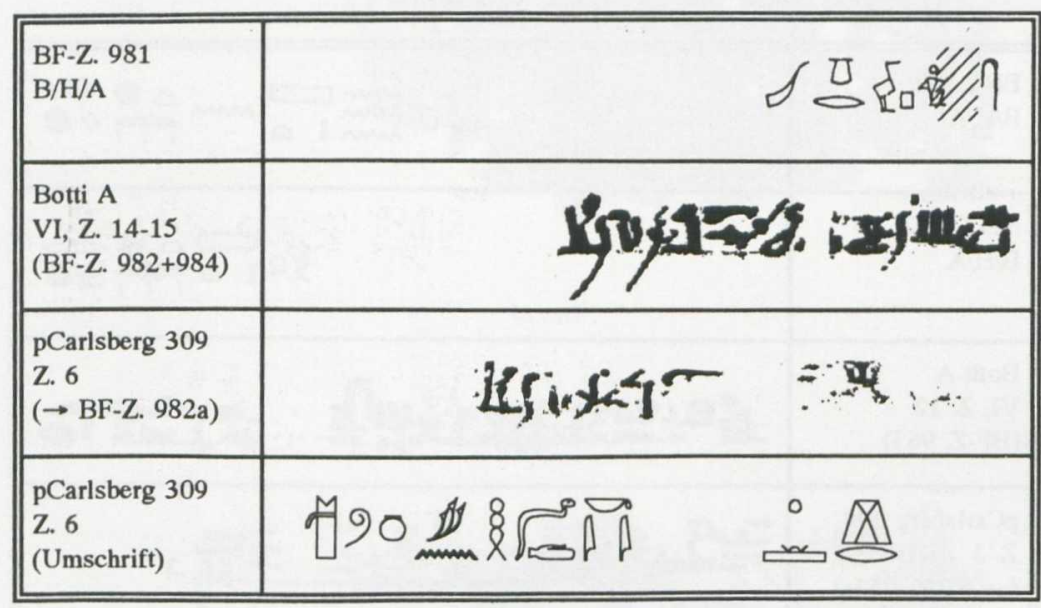

Abb. 31

Das Motto des Lebenshauses (BF-Zeile 986) ist auf dem Papyrusfragment leider nicht erhalten. Der Text (Z. 7) (s. Abb. 32) setzt erst wieder mit dem Schutz ein, den die vier geiergestaltigen Göttinnen dem Lebenshaus in den vier Himmelsrichtungen gewähren: „(... Nechbet ist es), die aus dem Norden kommet, Wadjet (ist es ....".

Zeile 8 (s. Abb. 33) bestätigt die Schreibung von Botti A (VI, 16), nach der das $s$ der hieroglyphischen Textversion (BF-Zeile 995) als $s w$ zu verstehen ist. Nach dem Inhalt kann man $s w$ nur als Reflexivum verstehen: (die Göttinnen) „breiten sich aus über die Lebenszeit seines (!) Re, ihres Vaters ...". Es scheint, daß der Text hier nicht richtig abgeschrieben ist, denn eine Nennung des Re mit Suffix ist wohl unmöglich. Wahrscheinlich ist die vermeintliche „Re-Hieroglyphe“ ursprünglich das Determinativ zu ' $h^{\complement}$ (Lebenszeit) gewesen, so wie wir es auch in der Handschrift Botti A finden. Dieses Determinativ fehlt ja auffälligerweise im Fragment pCarlsberg 309. Das $f$ hinter dem Determinativstrich von $r^{r}$ kann ich mir nicht erklären. Das $n$, das nach den beiden anderen Textversionen hier zu erwarten gewesen wäre, scheint bei unserem Fragment an `h` angehängt worden zu sein. Der Rest der Zeile entspricht dann wieder den beiden anderen Textzeugen.

Die 9. Zeile (s. Abb. 34) weicht wiederum von der bekannten Version (BF-Zeile 1001/1004) ab. Die Nennung der ,beiden Beine $(r d w j)$ “ in der Formulierung $h r r d w j$, die in der hieroglyphischen Version ganz deutlich ist (Taf. 28) und bei Botti A (VI, 17) über der Zeile nachgetragen ist, fehlt bei uns. Dafür finden wir vor dem Wort $\underline{h} r$ noch $\underline{h r}$ r.t.f. Das $f$ ist zwar nur in seiner obe- 


\begin{tabular}{|c|c|}
\hline $\begin{array}{l}\text { BF-Z. } 989 \\
\text { B/H/A }\end{array}$ & $\left\{a_{0}^{0}\left\{\gamma_{\Delta_{0}}^{\infty} \Rightarrow \|\right\}\right.$ \\
\hline $\begin{array}{l}\text { Botti A } \\
\text { VI, Z. } 15 \\
\text { (BF-Z. 990) }\end{array}$ & \\
\hline $\begin{array}{l}\text { pCarlsberg } 309 \\
\text { Z. } 7 \\
(\rightarrow \text { BF-Z. 990a) }\end{array}$ & iv \\
\hline $\begin{array}{l}\text { pCarlsberg } 309 \\
\text { Z. } 7 \\
\text { (Umschrift) }\end{array}$ & $a$ \\
\hline
\end{tabular}

Abb. 32

\begin{tabular}{|c|c|}
\hline $\begin{array}{l}\text { BF-Z. } 995 \\
\text { B/H/A }\end{array}$ & 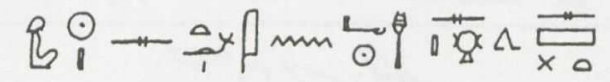 \\
\hline $\begin{array}{l}\text { Botti A } \\
\text { VI, Z. } 16 \\
\text { (BF-Z. } 996)\end{array}$ & -2 \\
\hline $\begin{array}{l}\text { pCarlsberg } 309 \\
\text { Z. } 8 \\
(\rightarrow \text { BF-Z. 996a) }\end{array}$ & 10 \\
\hline $\begin{array}{l}\text { pCarlsberg } 309 \\
\text { Z. } 8 \\
\text { (Umschrift) }\end{array}$ & 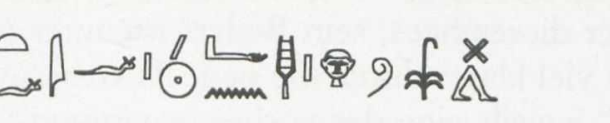 \\
\hline
\end{tabular}

Abb. 33

\begin{tabular}{|c|c|}
\hline $\begin{array}{l}\text { BF-Z. } 1001+1004 \\
\text { B/H/A }\end{array}$ & 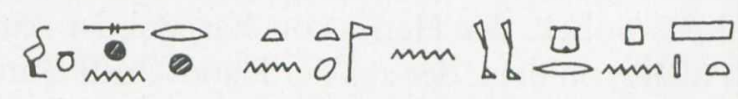 \\
\hline $\begin{array}{l}\text { Botti A } \\
\text { VI, Z. } 17 \\
(\text { BF-Z. } 1002+1005)\end{array}$ & \\
\hline $\begin{array}{l}\text { pCarlsberg } 309 \\
\text { Z. } 9 \\
(\rightarrow \text { BF-Z. 1002a) }\end{array}$ & IL ese: $x=$ \\
\hline $\begin{array}{l}\text { pCarlsberg } 309 \\
\text { Z. } 9 \\
\text { (Umschrift) }\end{array}$ & 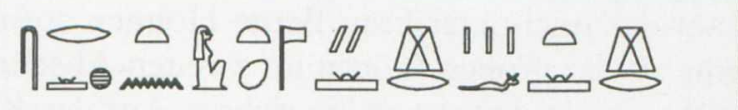 \\
\hline
\end{tabular}

Abb. 34 


\begin{tabular}{|c|c|}
\hline $\begin{array}{l}\text { Botti A } \\
\text { VI, Z. } 18 \\
\text { (BF-Z. 1010) }\end{array}$ & 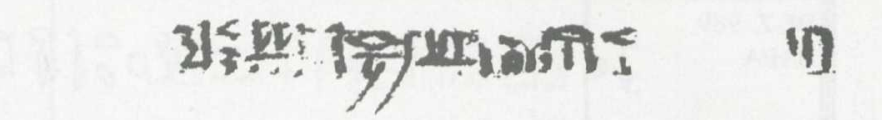 \\
\hline $\begin{array}{l}\text { pCarlsberg } 309 \\
\text { Z. } 10 \\
(\rightarrow \text { BF-Z. 1010a) }\end{array}$ & HIT I \\
\hline $\begin{array}{l}\text { pCarlsberg } 309 \\
\text { Z. } 10 \\
\text { (Umschrift) }\end{array}$ & III aلم \\
\hline
\end{tabular}

Abb. 35

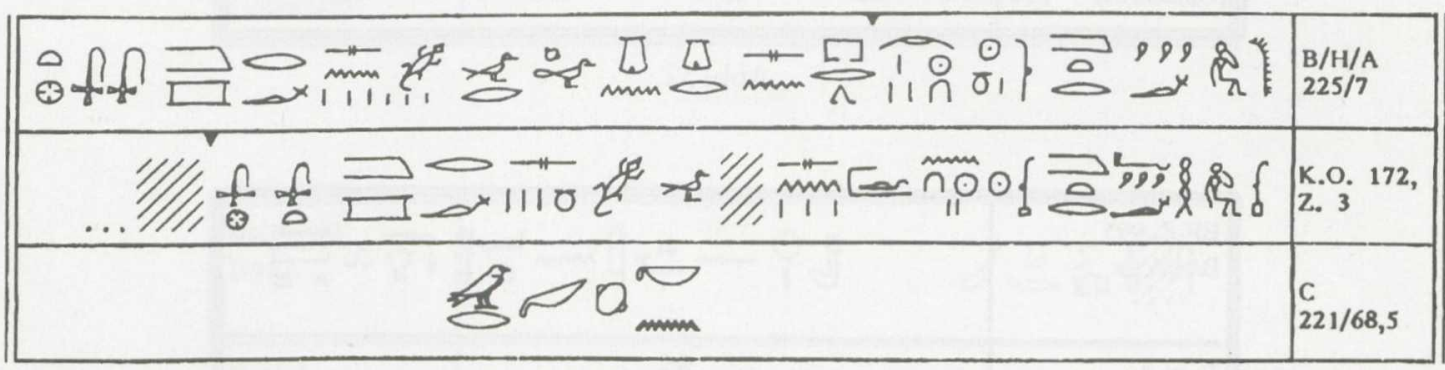

Abb. 36

ren Hälfte gut erhalten, m. E. aber trotzdem sicher zu erkennen. Da von den Worten am Anfang der Zeile nichts mehr lesbar ist, kann die Übersetzung nicht sicher sein. Ich würde vorschlagen, daß man das $f$ auf die in der BF-Zeile 1001 voranstehende Genitiv-Verbindung „Wadj-wer des Sees“ bezieht. Die Übersetzung der gesamten Zeile heißt dann: „Gegeben wird der (fruchtbare) Sand zum Wadj-wer dieses Sees, sein Bedarf ist unter (der Aufsicht) dieser Göttin." Inhaltlich wird der Text nicht viel klarer durch die neue Textvariante, und es muß weiter offen bleiben, ob mit „dieser Göttin“ jeweils eine der vorher genannten Schutzgöttinnen in Geiergestalt gemeint ist. Die abschließenden Worte der 9. Zeile leiten mit „rh - kennen“ die letzte Zeile des 5. Papyrusabschnittes ein, der noch in der Vignette steht: „Kennen jedes Suchen ...“.

Die unterste (10.) Zeile des pCarlsberg 309 (s. Abb. 35) ist eine Parallele zur BF-Zeile 1010. Es ist dies die einleitende Zeile des durchgängigen Textes des 5. Papyrusabschnittes. „(Dieser Platz) der Tempel des Sobek, des Herrn von Rasehui, ist sein Name. Die Felder ... "Der Text unterscheidet sich nicht von dem, der aus der Handschrift Botti A (VI, 18) bekannt ist.

\section{Papyrus Carlsberg 56 (BF-C), Ergänzung}

Obwohl der vorliegende Aufsatz eigentlich nur den hieratischen Nachträgen zum Text des „Buches vom Fayum“ vorbehalten sein sollte, benutze ich doch die Gelegenheit zu einer kleinen Ergänzung des hieroglyphischen „Buches vom Fayum“ in Kopenhagen. Als ich seinerzeit die diesbezüglichen Papyrusfragmente in Kopenhagen durchsehen durfte, ist mir ein kleines Fragment entgangen, auf das mich jetzt Frau Bente Holmen aufmerksam gemacht hat. Die Zuordnung des Fragments zu der großen Göttin im zweiten Abschnitt des „Buches vom Fayum“, die Frau Holmen vorgenommen hat, ist völlig richtig. Auf das Koordinatensystem übertragen, das ich im „Buch vom Fayum“ verwendet habe ${ }^{15}$, gehört das Fragment, wie sie durch den Verlauf

${ }^{15}$ Das Koordinatensystem ist auf der Tafel III nicht beigefügt, da die Tafel dadurch noch stärker verkleinert werden müßte, als es ohnehin der Fall ist. 

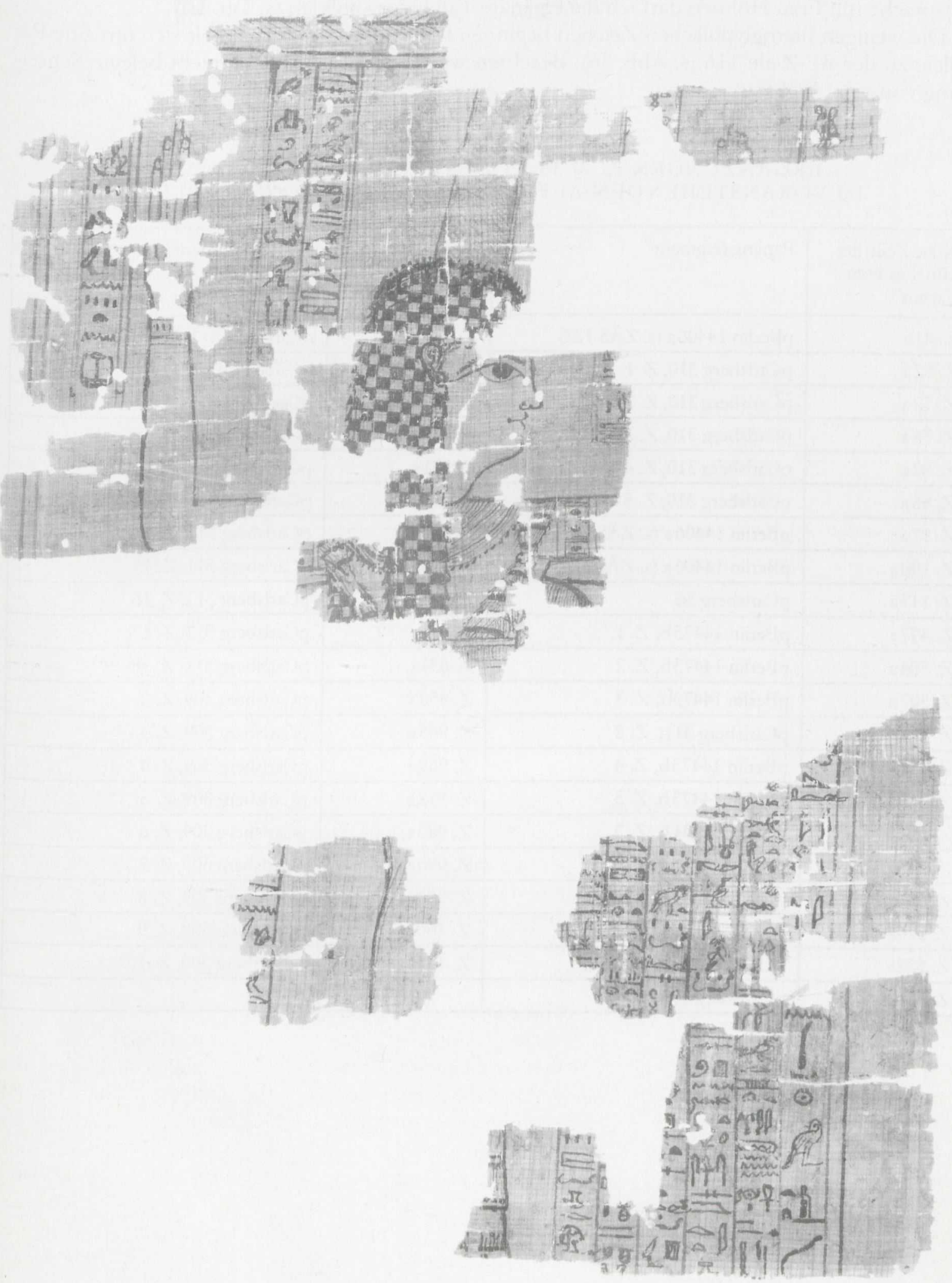

pCarlsberg 56 
der Papyrusfasern feststellen konnte, in das Rechteck aus $<219,8 / 68,1\rangle$ und $<224 / 72,9\rangle$. Nach Absprache mit Frau Holmen darf ich die ergänzte Tafel hier abbilden (s. Taf. III).

Die wenigen hieroglyphischen Zeichen beginnen bei $<221 / 68>$. Es handelt sich um eine $\mathrm{Pa}-$ rallele zu der BF-Zeile 116 (s. Abb. 36). Beachtenswert ist die bisher noch nicht belegte Schreibung von $q n-$ Arglist.

ERGÄNZUNGEN ZUM TEXT DES „BUCHES VOM FAYUM“

IM VORANSTEHENDEN AUFSATZ UND IN ZÄS 122 (ÜBERBLICK)

\begin{tabular}{|c|c|c|c|}
\hline $\begin{array}{l}\text { Neue Zeile des } \\
\text { „Buches vom } \\
\text { Fayum“ }\end{array}$ & Papyrusfragment & $\begin{array}{l}\text { Neue Zeile des } \\
\text { „Buches vom } \\
\text { Fayum“ }\end{array}$ & Papyrusfragment \\
\hline Z. $41 \mathrm{a}$ & pBerlin 14406 a (s. ZÄS 122) & Z. $550 \mathrm{a}$ & pCarlsberg 311, Z. 8 \\
\hline Z. $62 \mathrm{a}$ & pCarlsberg 310, Z. 1 & Z. $554 \mathrm{a}$ & pCarlsberg 311, Z. 9 \\
\hline Z. $72 \mathrm{a}$ & pCarlsberg 310, Z. 2 & Z. $560 \mathrm{a}$ & pCarlsberg 311, Z. 10 \\
\hline Z. $78 \mathrm{a}$ & pCarlsberg 310, Z. 3 & Z. $563 a$ & pCarlsberg 311, Z. 11 \\
\hline Z. $82 a$ & pCarlsberg 310, Z. 4 & Z. $571 \mathrm{a}$ & pCarlsberg 311, Z. 12 \\
\hline Z. $85 a$ & pCarlsberg 310, Z. 5 & Z. $580 \mathrm{a}$ & pCarlsberg 311, Z. 13 \\
\hline Z. $97 \mathrm{a}$ & pBerlin 14406a (s. ZÄS 122) & Z. $588 \mathrm{a}$ & pCarlsberg 311, Z. 14 \\
\hline Z. $100 \mathrm{a}$ & pBerlin 14406a (s. ZÄS 122) & Z. $595 \mathrm{a}$ & pCarlsberg 311, Z. 15 \\
\hline Z. $117 \mathrm{a}$ & pCarlsberg 56 & Z. $611 \mathrm{a}$ & pCarlsberg 311, Z. 16 \\
\hline Z. $497 a$ & pBerlin 14473b, Z. 1 & Z. $619 \mathrm{a}$ & pCarlsberg 311, Z. 17 \\
\hline Z. $504 \mathrm{a}$ & pBerlin 14473b, Z. 2 & Z. $631 \mathrm{a}$ & pCarlsberg 311, Z. 18 \\
\hline Z. $507 \mathrm{a}$ & pBerlin 14473b, Z. 3 & Z. $950 \mathrm{a}$ & pCarlsberg 309, Z. 2 \\
\hline Z. 507b & pCarlsberg 311, Z. 2 & Z. $963 \mathrm{a}$ & pCarlsberg 309, Z. 3 \\
\hline Z. 511a & pBerlin 14473b, Z. 4 & Z. $969 \mathrm{a}$ & pCarlsberg 309, Z. 4 \\
\hline Z. $515 \mathrm{a}$ & pBerlin 14473b, Z. 5 & Z. $978 \mathrm{a}$ & pCarlsberg 309, Z. 5 \\
\hline Z. $515 b$ & pCarlsberg 311, Z. 3 & Z. $982 \mathrm{a}$ & pCarlsberg 309, Z. 6 \\
\hline Z. $517 \mathrm{a}$ & pBerlin 14473b, Z. 6 & Z. $990 \mathrm{a}$ & pCarlsberg 309, Z. 7 \\
\hline Z. 521a & pCarlsberg 311, Z. 4 & Z. $996 a$ & pCarlsberg 309, Z. 8 \\
\hline Z. 526a & pCarlsberg 311, Z. 5 & Z. $1002 \mathrm{a}$ & pCarlsberg 309, Z. 9 \\
\hline Z. $532 \mathrm{a}$ & pCarlsberg 311, Z. 6 & Z. $1010 \mathrm{a}$ & pCarlsberg 309, Z. 10 \\
\hline Z. $542 \mathrm{a}$ & pCarlsberg 311, Z. 7 & & \\
\hline
\end{tabular}

